Article

\title{
Expression Profiling of Regulatory and Biosynthetic Genes in Contrastingly Anthocyanin Rich Strawberry (Fragaria $\times$ ananassa) Cultivars Reveals Key Genetic Determinants of Fruit Color
}

\author{
Mohammad Rashed Hossain ${ }^{1,2}$, Hoy-Taek Kim ${ }^{1,3, *}$, Ashokraj Shanmugam ${ }^{1}$, \\ Ujjal Kumar Nath ${ }^{1,2}$, Gayatri Goswami ${ }^{1}$, Jae-Young Song ${ }^{4}$, Jong-In Park ${ }^{1}$ and Ill-Sup Nou ${ }^{1, *}$ \\ 1 Department of Horticulture, Suncheon National University, 255 Jungang-ro, Suncheon, Jeonnam 57922, \\ Korea; m.r.hossain@bau.edu.bd (M.R.H.); araj866@gmail.com (A.S.); ujjalnath@gmail.com (U.K.N.); \\ gayatri_bau@yahoo.com (G.G.); jipark@sunchon.ac.kr (J.-I.P.) \\ 2 Department of Genetics and Plant Breeding, Bangladesh Agricultural University, \\ Mymensingh 2202, Bangladesh \\ 3 University-Industry Cooperation Foundation, Suncheon National University, 255 Jungang-ro, Suncheon, \\ Jeonnam 57922, Korea \\ 4 Department of Crop Science, Chungbuk National University, Chengju 28644, Korea; novaplant@cbnu.ac.kr \\ * Correspondence: htkim@sunchon.ac.kr (H.-T.K.); nis@sunchon.ac.kr (I.-S.N.); Tel.: +82-617-503-249 (I.-S.N.); \\ Fax: +82-617-505-389 (I.-S.N.)
}

Received: 27 January 2018; Accepted: 22 February 2018; Published: 26 February 2018

\begin{abstract}
Anthocyanins are the resultant end-point metabolites of phenylapropanoid/flavonoid (F/P) pathway which is regulated at transcriptional level via a series of structural genes. Identifying the key genes and their potential interactions can provide us with the clue for novel points of intervention for improvement of the trait in strawberry. We profiled the expressions of putative regulatory and biosynthetic genes of cultivated strawberry in three developmental and characteristically colored stages of fruits of contrastingly anthocyanin rich cultivars: Tokun, Maehyang and Soelhyang. Besides FaMYB10, a well-characterized positive regulator, FaMYB5, FabHLH3 and FabHLH3-delta might also act as potential positive regulators, while FaMYB11, FaMYB9, FabHLH33 and FaWD44-1 as potential negative regulators of anthocyanin biosynthesis in these high-anthocyanin cultivars. Among the early BGs, Fa4CL7, FaF3H, FaCHI1, FaCHI3, and FaCHS, and among the late BGs, FaDFR4-3, FaLDOX, and FaUFGT2 showed significantly higher expression in ripe fruits of high anthocyanin cultivars Maehyang and Soelhyang. Multivariate analysis revealed the association of these genes with total anthocyanins. Increasingly higher expressions of the key genes along the pathway indicates the progressive intensification of pathway flux leading to final higher accumulation of anthocyanins. Identification of these key genetic determinants of anthocyanin regulation and biosynthesis in Korean cultivars will be helpful in designing crop improvement programs.
\end{abstract}

Keywords: Fragaria $\times$ ananassa; anthocyanin; flavonoid; transcriptional regulation; biosynthetic genes

\section{Introduction}

Strawberry (Fragaria $\times$ ananassa) is favored across the globe not only due to its sweet-sour taste, unique flavors and rich nutritional values but also due to its attractive appearance [1]. These qualitative attributes are largely manifested by plant's inherent metabolic composition [2] where the flavonoid/phenylpropanoid $(\mathrm{F} / \mathrm{P})$ pathway plays key roles in determining the characteristic pigments of strawberry fruit [3-5]. The pigment is the resultant effect of accumulation of the secondary metabolites-anthocyanins-which play diverse roles ranging from fruit coloration and flavor to 
fruit ripening [6,7], increased pollination to seed dispersal via attracting pollinators and predators [8], anti-oxidative roles in protecting against UV light [9] and against abiotic and biotic stresses [5]. Besides, anthocyanins also have potential long-term health benefits including the production of health promoting compounds having anti-oxidative [10], anti-mutagenic and anticancer properties [11]. Anthocyanins thus play pivotal roles in consumer preference and marketability of strawberry fruits [12]. All these made anthocyanins and its biosynthetic pathway one of the well-studied pathways in strawberry and other fruits at genetic, biochemical and molecular levels $[5,10,13,14]$ and are therefore the targets of many strawberry breeding programs [3,4].

The pathway involves several enzymes that act in two stages, namely, early and late biosynthetic stages, leading to the biosynthesis of different flavonoids such as flavonols, condensed tannins and anthocyanidins which give rise to the characteristic colors in flowers and fruits of many species $[15,16]$. Early biosynthetic stages start with the catalysis of phenylalanine to yield cinnamic acid and coumaroyl-CoA followed by the synthesis of chalcone which is then isomerized to flavanone by the enzyme chalcone isomerase (CHI) [16]. The enzyme flavanone 3-hydroxylase $(\mathrm{F} 3 \mathrm{H})$ catalyzes flavanones to dihydroflavonols. In late biosynthetic steps, dihydroflavonol reductase (DFR) reduces dihydroflavonols to leucoanthocyanins which in turn is converted to anthocyanidins by leucoanthocyanidin dioxygenase/anthocyanidin synthase (LDOX/ANS). The anthocyanidins are finally glycosylated to anthocyanins via the enzyme uridine diphosphate (UDP)-glucose:flavonoid-O-glycosyl-transferase (UFGT) [5,17].

These structural genes involved in the biosynthesis of anthocyanins are mainly regulated at transcriptional level via a ternary regulatory $M Y B-b H L H-W D 40$ " $M B W^{\prime}$ " protein complex which is formed by highly conserved transcription factors (TFs) R2R3-MYB interacting or not with MYC-like basic helix-loop-helix $(b H L H)$ proteins and/or with WD40-repeat proteins [12,18]. The TFs of the $M B W$ complex is identified in many species including Arabidopsis [19], grapevine [20], tomato and pepper [21], maize [22], potato [23], apple [24,25], sweet cheery [26,27], etc. The $M B W$ complex is unique to plants, varies in monocot vs. dicot species, and not all three members of the complex are needed for anthocyanin biosynthesis in every species [16,17]. For example, in maize (monocot), TFs of $M B W$ complex activates the biosynthesis of anthocyanin as a single unit [28]. In Arabidopsis, the early biosynthetic genes can be regulated by an independent $R 2 R 3-M Y B$ co-activator while late biosynthetic genes are known to be regulated by ternary $M B W$ complex [16]. In grape and apple, MYB-bHLH complex (lacking WD40) regulates anthocynin biosynthesis [25]. In strawberry, FaMYB10 is reported as the key regulator of anthocyanin biosynthesis [29]. Besides these activators, few TFs may act as negative regulator of the pathway in different species such as FaMYB1 and FcMYB1 in strawberry [30,31]; MdMYB16, MdMYB17 and MdMYB111 in apple [32]; and AtMYB3, AtMYB4 and AtMYBL2 in Arabidopsis [33].

The combinatorial interactions of the regulatory genes activate or inhibit the expression of individual or set of structural genes (some of which might have pleiotropic effects) that act in a coordinated manner to control the flux of various branches of the pathway which ultimately determines the flavonoid composition in the tissue $[3,12,15]$. The pathway is also modulated by environmental factors, especially light and temperature, which further influence the qualitative and quantitative composition of anthocyanins [34-36].

Thus, studying the cultivar specific regulatory, early- and late-biosynthetic genes together will be helpful in identifying the overall association of the genes involved and the key steps of the anthocyanin biosynthetic pathway which can enable us to target specific gene(s) and develop molecular markers for breeding programs for improving pigmentation in strawberry. The current study thus investigates the key genetic determinants of anthocyanin accumulation in three popular contrastingly pigmented strawberry cultivars of Korea by associating the expressions of all regulatory, early- and late-biosynthetic genes with the accumulation of anthocyanins in strawberry fruits based on a multivariate statistics based approach. 


\section{Results}

\subsection{Total Anthocyanin Varied Across Fruit Developmental Stages and Cultivars of Strawberry}

In general, total anthocyanin contents varied significantly $(p<0.01)$ across the fruit developmental stages and were higher in ripe fruits followed by green and white fruits in all three cultivars (Figure 1B). A generalized decrease in the total anthocyanin contents in white stage; a less pigmented transitional stage between green and ripe stages were observed in all three cultivars. Notable was that the ripe fruits of Maehyang and Soelhyang contained significantly higher amount of total anthocyanin (approximately six- and five-fold higher, respectively, compared to that of the ripe fruits of Tokun) which was apparent from the visual observations of the ripe fruits and the tubes containing anthocyanin extract as well (Figure 1A,B). This clearly indicates Maehyang and Soelhyang as high anthocyanin containing cultivars compared to Tokun based on the content of anthocyanin in ripe fruits in particular.

A

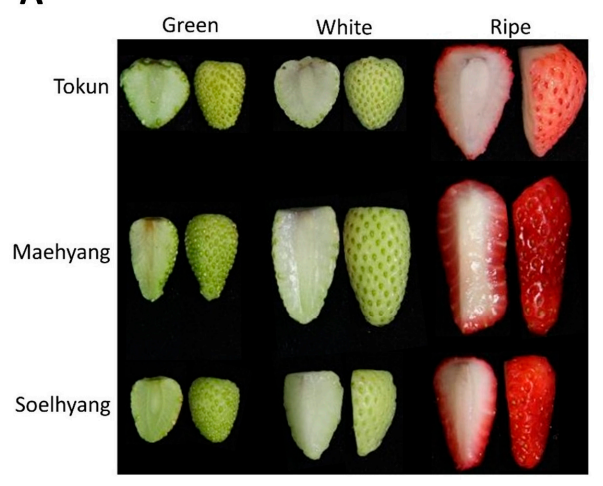

B

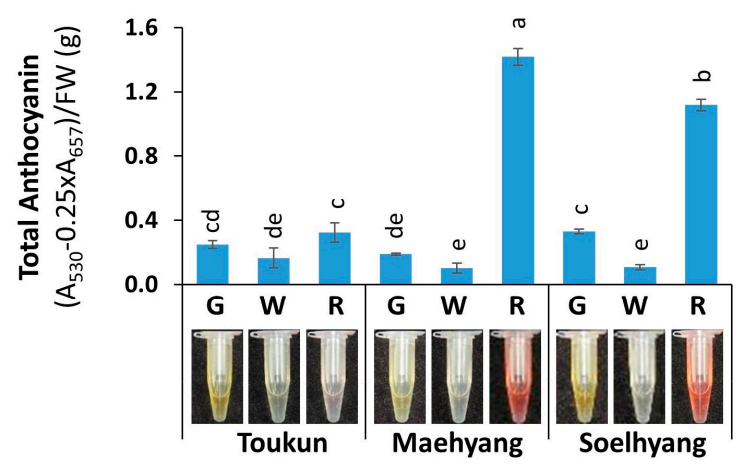

Figure 1. Strawberry (Fragaria $\times$ ananassa) fruits $(\mathbf{A})$; and contents of total anthocyanin $(\mathbf{B})$ in three developmental stages of fruits, namely, green, white and ripe stages of cultivar, Tokun, Maehyang and Soelhyang are used in this study. Data are presented as mean $\pm \mathrm{SD}(n=3)$. Fruit developmental stages of the cultivars varied significantly $(p<0.01)$ for total anthocyanin as determined by one-way ANOVA. Statistically significant differences in the total anthocyanin content are indicated by different letters as per Tukey's pairwise comparisons. G, Green; W, White; R, Ripe.

\subsection{FaMYB10 Dominated the Anthocyanin Regulatory Complex}

Highly variable expressions were obtained for the studied 14 regulatory genes of $M B W$ protein complex in the three developmental stages of fruits of three differentially anthocyanin containing cultivars. Among the MYB transcription factors (TFs), FaMYB10, a well-known MYB TF, showed remarkable higher expressions in the ripe fruits of high anthocyanin containing cultivars, Maehyang and Soelhyang (Figure 2). Compared to the expressions of the green fruits of low anthocyanin containing cultivar Tokun, the gene expressions were $\sim 21$ - and 37-fold higher, respectively ( 2- and $\sim 3$-fold higher than the ripe fruits of Tokun, respectively). The gene FaMYB5 showed slightly increased statistically significant expressions in the ripe fruits of Maehyang and Soelhyang compared to the respective green fruits while its expressions remained unchanged in all three stages of the cultivar Tokun. Expression of FaMYB1 significantly increased only in the ripe fruits of Soelhyang compared to initial developmental stages of fruits while it remained unchanged in the other high anthocyanin containing cultivar Maehyang. Another volatile related MYB TF, FaEOBII (GENE28435), also showed significant increase in the ripe fruits of Maehyang and Soelhyang but its expression was also increased in the low anthocyanin containing cultivar Tokun.

Among the four bHLH genes, FabHLH3 and FabHLH3-delta showed significantly increased expressions in the ripe fruits of Maehyang and Soelhyang compared to that of the green fruits, respectively, while its expression in the fruits of Tokun remained statistically unchanged (Figure 2). Of these, only the FabHLH3, however, exhibited significant positive correlation $(p<0.05)$ with FaMYB10 
(Table S1). Among the WD40 protein coding genes, only the expressions of FaTTG1 were found to be significantly higher in the white and ripe fruits of Soelhyang while the expression of the other three genes were either decreased or statistically similar in both low and high anthocyanin cultivars. Taken together, it is apparent that FaMYB10 dominated the regulatory complex with approximately 20- and 36-fold higher expressions, along with the FabHLH3 and FabHLH3-delta genes, which showed approximately 3-4 times higher expression in ripe fruits of Maehyang and Soelhyang compared to that of the green fruits of low anthocyanin cultivar Tokun.
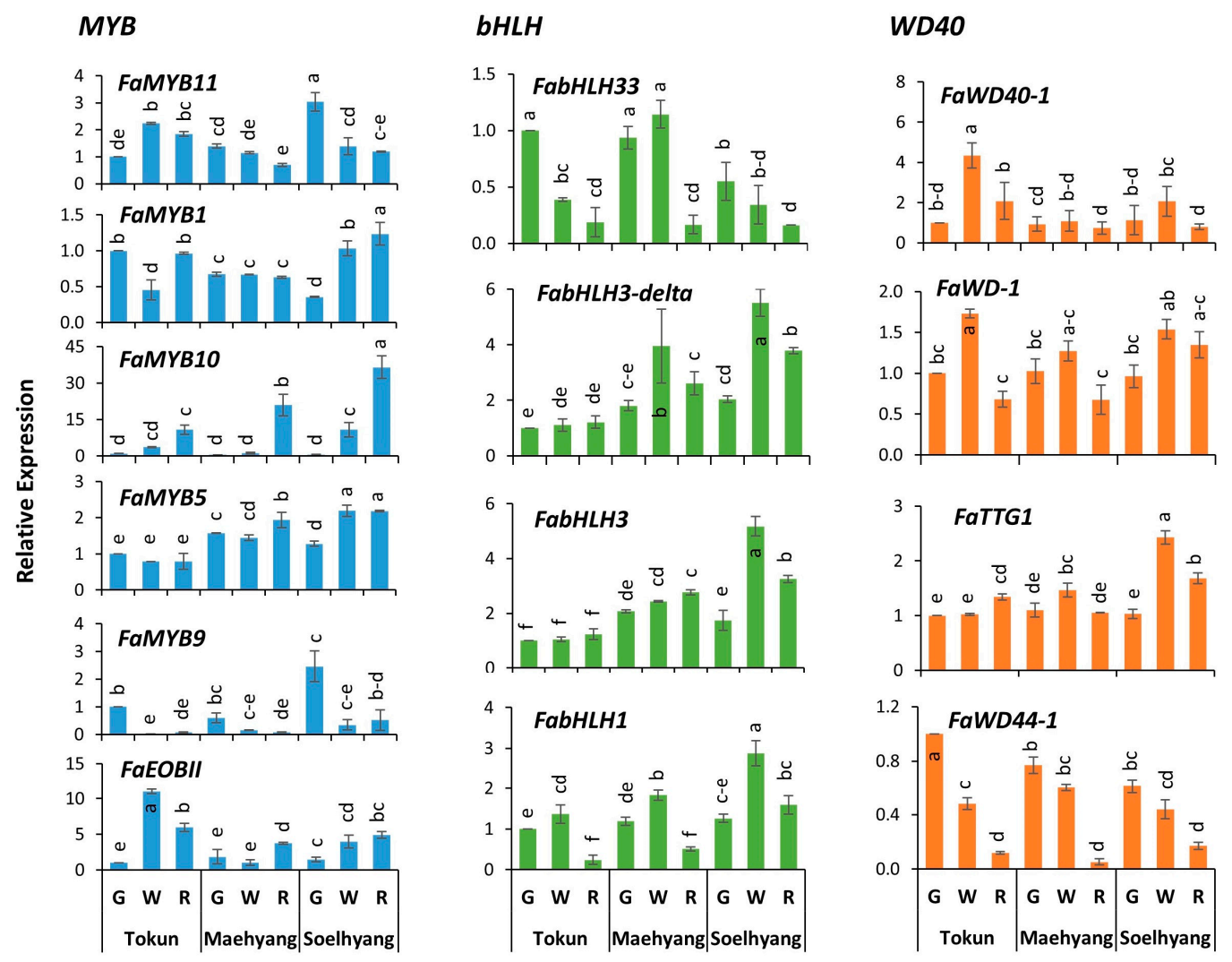

Figure 2. Expression analysis of the regulatory genes involved in the biosynthesis of anthocyanin by quantitative real-time PCR in the three fruit developmental stages, namely, green, white and ripe fruits of Fragaria $\times$ ananassa fruit. Data are presented as mean $\pm \mathrm{SD}(n=3)$. Fruit developmental stages of the cultivars varied significantly $(p<0.01)$ for relative expressions as determined by one-way ANOVA. Statistically significant differences in the relative expressions are indicated by different letters as per Tukey's pairwise comparisons. G, Green; W, White; R, Ripe.

\subsection{FaMYB11 Is the Potential Negative Regulator of Anthocyanin Biosynthesis}

Among the MYB TFs, the expressions of FaMYB11 were decreased with the progressive developmental stages of fruits in high anthocyanin containing cultivars Maehyang and Soelhyang (decreased by approximately two and three folds compared to the respective green fruits, respectively) while its expression was significantly increased in low anthocyanin cultivar Tokun (Figure 2). This contrasting pattern of expression in high and low anthocyanin cultivars makes this gene the potential repressor of anthocyanin biosynthesis. Expressions of another MYB, FaMYB9, were decreased in both high and low anthocyanin cultivars (approximately 5-7 and 15 times, respectively) compared to the respective green fruits. Among the bHLH TFs, expressions of FabHLH33 were also decreased (by approximately 3-6 folds) significantly in ripe fruits of all three cultivars. Such general decrease in expression (by approximately 4-15 folds) was also observed for FaWD44-1 in all three cultivars which showed significant negative correlation with FaMYB10 $(p<0.001$; Table S1), the key positive regulator 
of anthocyanin biosynthesis. The expression of FabHLH1, FaWD40-1 and FaWD-1 showed an increase in white stage before being decreased in the ripe fruits of all three cultivars (Figure 2); a pattern which needs further investigation to determine their specific roles.

\subsection{Expression Profiles of Early Biosynthetic Genes}

Expressions of the structural genes involved in the major metabolic steps of the anthocyanin biosynthesis were studied and significant variations were obtained across the fruit developmental stages and across contrastingly anthocyanin rich cultivars. Among the genes involved in early biosynthetic steps (phenylalanine to flavanone), the genes of the first two steps, namely, phenylalanine and cinnamic acid, did not show any notable higher expressions except phenylalanine ammonia lyase gene FaPAL2, which showed a two-fold higher expression in ripe fruits of Soelhyang (compared to green fruits of Tokun) only. However, the expressions of phenylalanine gene FaPAL1 and cinnamate-4-hydroxylase gene $\mathrm{FaC} 4 \mathrm{H}$ were significantly decreased in the ripe fruits of low anthocyanin cultivar Tokun (compared to green fruits) while their expressions remained somewhat unchanged in the high anthocyanin cultivars, Maehyang and Soelhyang (Figure 3).

The expression of Coumaroyl-CoA ligase gene Fa4CL7 were significantly increased from green to ripe fruits in all three cultivars. However, the increase was much higher in the high anthocyanin cultivars Maehyang ( $\sim 2$ folds) and Soelhyang ( 17 folds) compared to that of low anthocyanin cultivar Tokun (only $\sim 5$ folds). This indicates its potential role in overall anthocyanin biosynthesis. The expression of the other Coumaroyl-CoA ligase gene, Fa4CL2, showed $~ 7$ - and $~ 9$-fold decreases in the ripe fruits compared to green fruits of high anthocyanin cultivars Maehyang and Soelhyang, respectively, but remained unchanged in low anthocyanin cultivar Tokun (Figure 3).

Phenylalanine

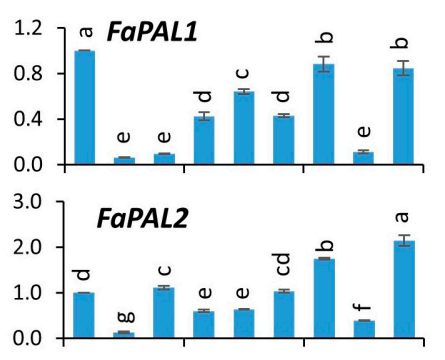

Cinnamic acid

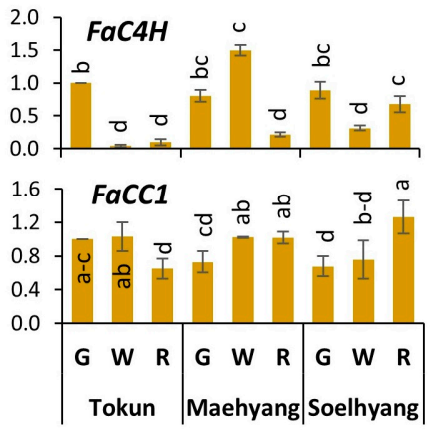

Coumaroyl-CoA

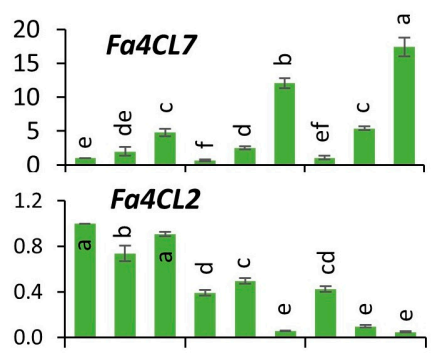

Flavanone

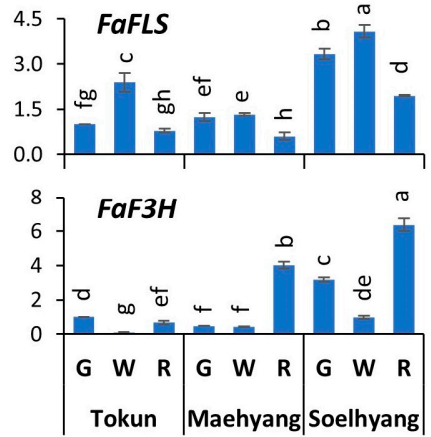

\section{Chalcone}

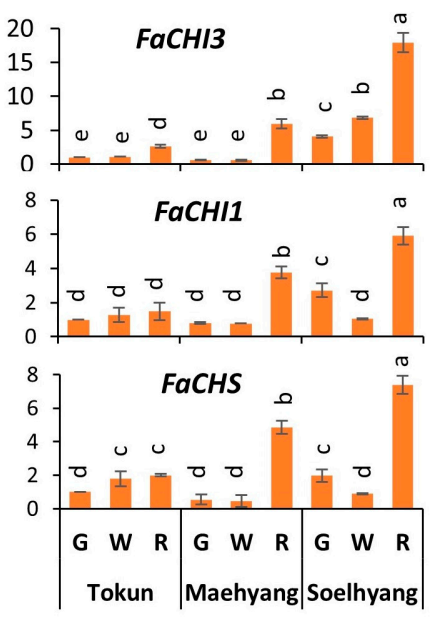

Figure 3. Expression analysis of the genes involved in the early biosynthetic steps of anthocyanin biosynthetic pathway by quantitative real-time PCR in the three fruit developmental stages, namely, green, white and ripe fruits, of Fragaria $\times$ ananassa fruit. Data are presented as mean $\pm \mathrm{SD}(n=3)$. Fruit developmental stages of the cultivars varied significantly $(p<0.01)$ for relative expressions as determined by one-way ANOVA. Statistically significant differences in the relative expressions are indicated by different letters as per Tukey's pairwise comparisons. G, Green; W, White; R, Ripe. 
Among the genes involved in Chalcone synthesis, both the Chalcon Isomerase genes, $\mathrm{FaCHII}$ and $\mathrm{FaCHI3}$, and Chalcon Synthase gene, $\mathrm{FaCHS}$, showed much higher expressions in the ripe fruits of high anthocyanin cultivars Maehyang and Soelhyang compared to that of the low anthocyanin cultivar Tokun, indicating the importance of this step (and the genes involved) in overall anthocyanin biosynthesis. For example, $\mathrm{FaCHI} 3$ was $\sim 10$ - and $\sim 4$-fold higher expressed in Maehyang and Soelhyang, respectively, while it was only expressed $\sim 2$ folds in Tokun.

Among the genes involved in Flavanone biosynthesis, flavanone 3 hydroxylase $(\mathrm{FaF} 3 \mathrm{H})$ was expressed by $\sim 8$ and $\sim 2$ folds in the ripe fruits of Maehyang and Soelhyang, respectively, compared to respective green fruits, while its expression did not show such increase in Tokun ripe fruits. Flavonol synthase on the other hand showed general decrease in the ripe fruits of all three cultivars.

\subsection{Key Genes of the Late Biosynthetic Steps}

Higher expressions were observed for the genes involved in each of the late biosynthetic steps (Figure 4). Among the dihydroflavanol reductase genes, FaDFR4-3 showed increasing trend of expression from green to ripened stages of fruits in all three cultivars. However, the expressions were much higher in the ripe fruits of high anthocyanin cultivars, Maehyang and Soelhyang ( $\sim 9$ and $\sim 24$ folds, respectively) compared to only 2.29-fold increase in the low anthocyanin cultivar Tokun (Figure 4). The expressions of the other two genes, FaDFR4-1 and FaDFR4-2, did not show such contrastingly increasing patterns of expression between high and low anthocyanin cultivars. For these two genes, the expressions were similar in green and ripe fruits, but, interestingly, a general increase in the intermediate white stage is observed for each of the three cultivars.

\section{Dihydroflavonol}

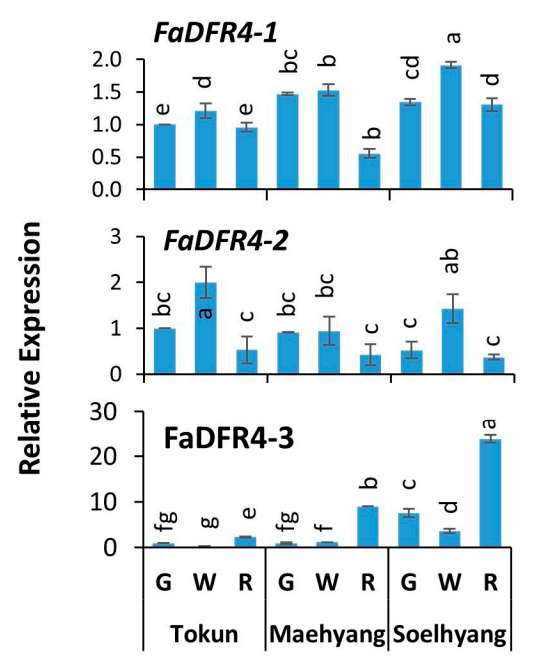

\section{Leucoanthocyanidin}
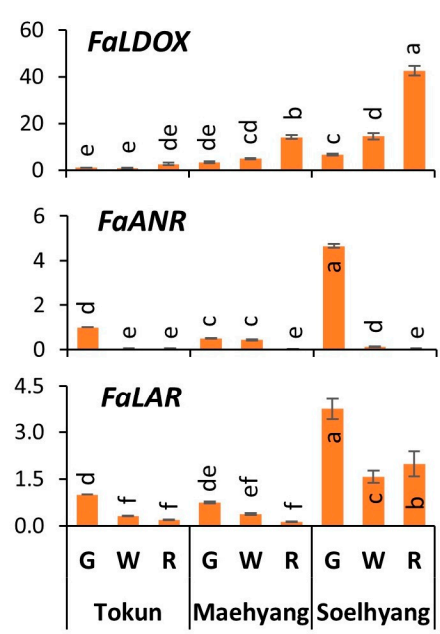

\section{Anthocyanidin}
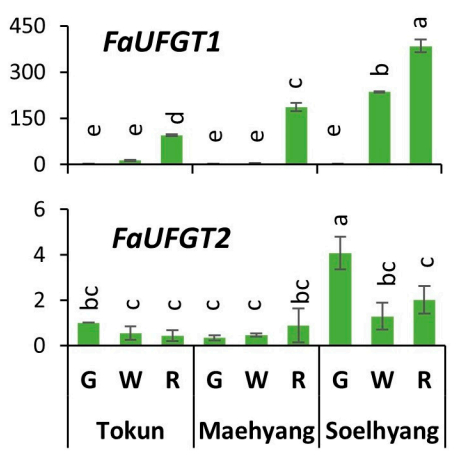

Figure 4. Expression analysis of the genes involved in the late biosynthetic steps of anthocyanin biosynthetic pathway by quantitative real-time PCR in the three fruit developmental stages, namely, green, white and ripe fruits, of Fragaria $\times$ ananassa fruit. Data are presented as mean $\pm \operatorname{SD}(n=3)$. Fruit developmental stages of the cultivars varied significantly $(p<0.01)$ for relative expressions as determined by one-way ANOVA. Statistically significant differences in the relative expressions are indicated by different letters as per Tukey's pairwise comparisons. G, Green; W, White; R, Ripe.

Among the genes involved in the leucoanthocyanidin step, leucoanthocyanidin dioxygenase FaLDOX showed increasing pattern of expression from green to ripened stages of fruits in all three cultivars. In the ripe fruits of high anthocyanin cultivars, Maehyang and Soelhyang, the gene showed $\sim 14$ - and $\sim 42$-fold higher expressions compared to the green fruits of low anthocyanin cultivar Tokun (four and six folds compared to respective green fruits) (Figure 4). The other two genes, namely, 
Anthocyanidin reductase ( $F a A N R)$ and Leucoanthocyanidin reductase ( $F a L A R)$, showed decreasing patterns of expression in all three cultivars. Anthocyanidin reductase ( $F a A N R)$, in particular, was almost not expressed in the ripe fruits, while the green fruits showed significantly high expressions indicating a possible repressing role played by this genes in overall anthocyanin biosynthesis pathway.

The most striking increasing pattern of expression was obtained for the uridine diphosphate-glucose:flavonoid 3-O-glucosyltransferase gene, FaUFGT1, involved in the very final step of anthocyanin biosynthetic pathway, showing around 123- and 313-fold higher expressions (compared to the respective green fruits) in the ripe fruits of high anthocyanin cultivars, Maehyang and Soelhyang (Figure 4).

\subsection{Late Biosynthetic Genes Show Comparatively Higher Expression}

A general notion is observed that, compared to the expressions of the early biosynthetic genes, late biosynthetic genes showed higher expressions in the ripe fruits of high anthocyanin cultivars, Maehyang and Soelhyang. For example, the highest relative expressions observed for early biosynthetic genes were 17.90 ( $\mathrm{FaCHI}$ ); 17.41 ( $\mathrm{Fa} 4 \mathrm{CL} 7$ ) in the ripe fruits of high anthocyanin cultivar Soelhyang compared to the green fruits of low anthocyanin cultivar Tokun (Figure 3). Few of the late biosynthetic genes showed much higher expressions such as FaDFR4-3 (23.84), FaLDOX (42.48) and FaUFGT1 (384.29) in the ripe fruits of high anthocyanin cultivar Soelhyang (compared to the green fruits of low anthocyanin cultivar Tokun) (Figure 4). This probably indicates much greater role of the final steps of the anthocyanin biosynthesis pathways (and the genes involved in those steps) in final accumulation of anthocyanin in ripe fruits which give rise to the characteristic color in ripe fruits.

\subsection{Association between Contents of Total Anthocyanin and Expressions of Related Regulatory and Biosynthetic Genes}

Principal Component Analysis (PCA) of the contents of total anthocyanin, the expressions of regulatory and biosynthetic genes in three developmental stages of fruit ripening in contrasting cultivars extracted six principal components (PCs) having eigenvalue greater than unity (data not shown). The first three PCs explained $73.5 \%$ of the total variation in the entire datasets. PC1 accounted for $36.6 \%$ of the total variation which is mainly manifested by the higher positive coefficients of total anthocyanins (0.23), MYB10 (0.26), Fa4CL7 (0.27), FaF3H (0.26), FaCHI3 (0.27), FaCHI1 (0.25), FaCHS (0.26), FaDFR3 (0.26) and FaUFGT1 (0.26) versus higher negative coefficients of FabHLH33 (-0.173), FaWD40-1 (-0.104), FaWD44-1 (-0.176), Fa4CL2 (-0.204), FaDFR-2 (-0.136) and MYB11 (-0.080) (Table 1 and Figure 5). PC1 clearly distinguished the key highly expressed genes from the rest and the anthocyanin rich ripe fruits of Maehyang and Soelhyang from the other less anthocyanin containing samples as evident by their mean PC scores in opposite direction in these contrasting samples and differential positioning in PCA biplot (Table 1 and Figure 5). The highly expressed genes were plotted along the total anthocyanin content and ripe fruit samples of high anthocyanin cultivars, Maehyang and Soelhyang as shown in PCA biplot (Figure 5). This is also corroborated by the higher significant positive correlations between total anthocyanin and these genes, as observed from the Pearson's correlation analysis (Table S1). 
Table 1. Component loadings and mean PC scores showing the association between total anthocyanin and expressions of regulatory and biosynthetic genes as determined by Principal Component Analysis. PC, Principal Component; p, statistical significance; SD, Standard Deviation.

\begin{tabular}{|c|c|c|c|c|}
\hline \multicolumn{2}{|c|}{ Variable } & PC1 & PC2 & PC3 \\
\hline \multicolumn{5}{|c|}{ Regulatory Genes } \\
\hline \multirow{6}{*}{$M Y B$} & FaMYB11 & -0.080 & 0.128 & -0.115 \\
\hline & FaMYB1 & 0.132 & -0.020 & 0.139 \\
\hline & FaMYB10 & 0.260 & -0.094 & 0.044 \\
\hline & FaMYB5 & 0.205 & 0.146 & 0.131 \\
\hline & FаМҮB9 & -0.012 & 0.253 & -0.271 \\
\hline & FaEOBII & 0.011 & -0.216 & 0.151 \\
\hline \multirow{4}{*}{$b H L H$} & FabHLH33 & -0.173 & 0.142 & -0.043 \\
\hline & FabHLH3-delta & 0.128 & 0.192 & 0.232 \\
\hline & FabHLH3 & 0.148 & 0.170 & 0.238 \\
\hline & FabHLH1 & 0.018 & 0.271 & 0.267 \\
\hline \multirow{4}{*}{ WD40 } & FaWD40-1 & -0.104 & -0.110 & 0.166 \\
\hline & FaWD-1 & -0.033 & 0.068 & 0.239 \\
\hline & FaTTG1 & 0.108 & 0.154 & 0.285 \\
\hline & FaWD44-1 & -0.176 & 0.198 & -0.066 \\
\hline \multicolumn{5}{|c|}{ Early Biosynthetic Genes } \\
\hline \multirow{2}{*}{ Phenylalanine } & FaPAL1 & 0.064 & 0.171 & -0.264 \\
\hline & FaPAL2 & 0.189 & 0.078 & -0.254 \\
\hline \multirow{2}{*}{ Cinnamic acid } & $\mathrm{FaC} 4 \mathrm{H}$ & -0.047 & 0.228 & -0.113 \\
\hline & $\mathrm{FaCC1}$ & 0.143 & -0.059 & 0.026 \\
\hline \multirow{2}{*}{ Coumaroyl CoA } & Fa4CL7 & 0.268 & -0.094 & 0.036 \\
\hline & Fa4CL2 & -0.204 & -0.116 & -0.104 \\
\hline \multirow{3}{*}{ Chalcone } & $\mathrm{FaCHI3}$ & 0.266 & 0.051 & 0.022 \\
\hline & $\mathrm{FaCHI1}$ & 0.254 & -0.014 & -0.121 \\
\hline & $\mathrm{FaCHS}$ & 0.256 & -0.100 & -0.074 \\
\hline \multirow{2}{*}{ Flavanone } & FaFLS & 0.018 & 0.273 & 0.134 \\
\hline & $\mathrm{FaF3H}$ & 0.258 & 0.028 & -0.137 \\
\hline \multicolumn{5}{|c|}{ Late Biosynthetic Genes } \\
\hline \multirow{3}{*}{ Dihydroflavonol } & FaDFR4-1 & -0.035 & 0.294 & 0.222 \\
\hline & FaDFR4-2 & -0.136 & 0.014 & 0.216 \\
\hline & FaDFR4-3 & 0.264 & 0.042 & -0.076 \\
\hline \multirow{3}{*}{ Leucoanthocyanidin } & FaLDOX & 0.266 & 0.059 & 0.037 \\
\hline & FaANR & -0.049 & 0.233 & -0.265 \\
\hline & FaLAR & 0.074 & 0.300 & -0.157 \\
\hline \multirow{2}{*}{ Anthocyanidin } & FaUFGT1 & 0.260 & -0.015 & 0.126 \\
\hline & FaUFGT2 & 0.082 & 0.245 & -0.192 \\
\hline \multicolumn{2}{|c|}{ Total anthocyanin } & 0.229 & -0.133 & -0.110 \\
\hline \multicolumn{2}{|c|}{ Eigenvalue } & 12.809 & 6.630 & 6.280 \\
\hline \multirow{2}{*}{\multicolumn{2}{|c|}{$\begin{array}{c}\text { \% variation explained } \\
\text { P (Genotype } \times \text { Fruit developmental stage })\end{array}$}} & 36.6 & 18.9 & 17.9 \\
\hline & & $<0.001$ & $<0.001$ & $<0.001$ \\
\hline \multicolumn{2}{|c|}{ Genotype $\times$ Fruit Developmental Stage } & \multicolumn{3}{|c|}{ Mean PC Scores \pm SD } \\
\hline \multirow{3}{*}{ Tokun } & Green & $-2.75 \pm 0.01 \mathrm{~g}$ & $-0.06 \pm 0.01 \mathrm{~d}$ & $\begin{array}{c}-2.08 \pm 0.01 \\
\mathrm{e}\end{array}$ \\
\hline & White & $-3.15 \pm 0.37 \mathrm{~g}$ & $-2.48 \pm 0.02 \mathrm{e}$ & $1.87 \pm 0.10 \mathrm{~b}$ \\
\hline & Ripe & $-1.23 \pm 0.14 \mathrm{e}$ & $-3.51 \pm 0.08 \mathrm{f}$ & $\begin{array}{c}-0.45 \pm 0.12 \\
\mathrm{~cd}\end{array}$ \\
\hline & Green & $-2.64 \pm 0.17 \mathrm{fg}$ & $0.55 \pm 0.26 \mathrm{~d}$ & $0.04 \pm 0.13 \mathrm{c}$ \\
\hline Maehyang & White & $-2.18 \pm 0.30 \mathrm{f}$ & $1.54 \pm 0.02 \mathrm{c}$ & $1.11 \pm 0.16 \mathrm{~b}$ \\
\hline & Ripe & $3.57 \pm 0.06 b$ & $-3.44 \pm 0.04 \mathrm{f}$ & $\begin{array}{c}-1.10 \pm 0.02 \\
\text { de }\end{array}$ \\
\hline & Green & $-0.66 \pm 0.19 \mathrm{~d}$ & $3.94 \pm 0.55 \mathrm{a}$ & $\begin{array}{c}-4.26 \pm 0.41 \\
\mathrm{f}\end{array}$ \\
\hline Soelhyang & White & $0.89 \pm 0.13 c$ & $2.88 \pm 0.30 b$ & $5.01 \pm 0.67 \mathrm{a}$ \\
\hline & Ripe & $8.14 \pm 0.02 \mathrm{a}$ & $0.588 \pm 0.36 \mathrm{~d}$ & $\begin{array}{c}-0.14 \pm 0.64 \\
\mathrm{~cd}\end{array}$ \\
\hline
\end{tabular}

$P A L$, Phenylalanine ammonia lyase; $C 4 H$, cinnamate-4-hydroxylase; $4 C L$, 4-coumaroyl-CoA-ligase; $C H S$, Chalcone synthase; $C H I$, Chalcone isomerase; $F 3 H$, Flavanone 3 hydroxylase; $F L S$, Flavonol synthase; DFR, Dihydroflavanol reductase; $L A R$, Leucoanthocyanidin reductase; $A N R$, Anthocyanidin reductase; $L D O X$, leucoanthocyanidin dioxygenase; UFGT, uridine diphosphate-glucose:flavonoid 3-O-glucosyltransferase. Statistically significant differences in the relative expressions are indicated by different letters as per Tukey's pairwise comparisons. 


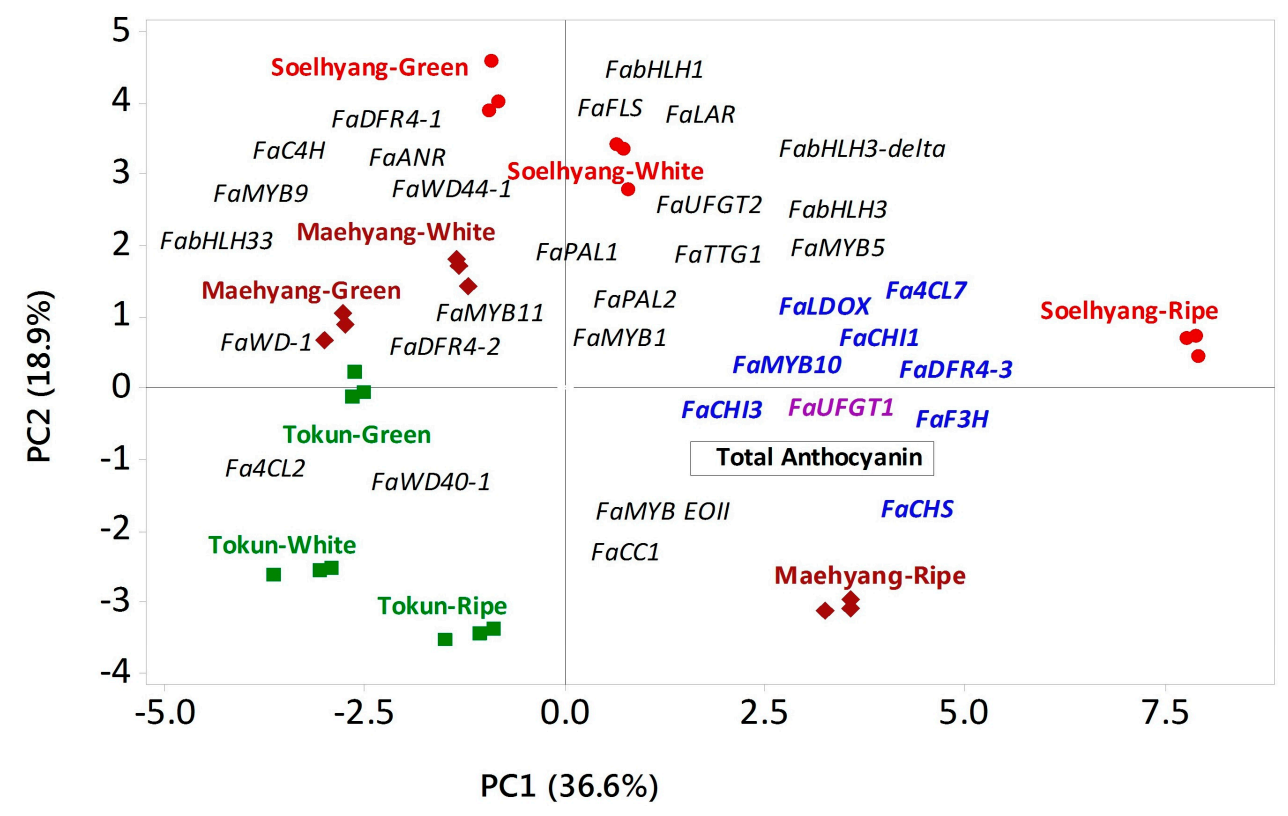

Figure 5. Biplot showing the association between the regulatory and biosynthetic genes with the total anthocyanin in three fruit developmental stages, namely, green, white and ripe fruits, of Fragaria $\times$ ananassa fruit as determined by Principal Component Analysis (PCA). The three varieties are shown in three different shapes and colors as positioned by their respective mean PC scores in PCA biplot. The total anthocyanin (shown in rectangular box) and the genes represent coefficients between PC1 and PC2. The most contributing genes in accumulating higher anthocyanin in ripe fruits of cultivars, Maehyang and Soelhyang, are shown by bold letters. PC, Principal Component.

\section{Discussion}

Anthocyanins are plant's secondary metabolites that render the attractive pigmentation and characteristic flavor to many fruits along with potential health benefits to human. This study attempted to identify the key regulatory and structural genes and their association with total anthocyanins in contrastingly pigmented strawberry cultivars.

\subsection{Positive Regulators of Anthocyanin Biosynthesis in Korean Strawberry Cultivars}

Expression analysis of the 14 selected regulatory genes in the fruits of contrasting cultivars identified FaMYB10 as the most highly expressed and FaMYB5, FabHLH3 and FabHLH3-delta as somewhat ( $\sim-4$ folds) highly expressed TFs in high anthocyanin containing fruits (Figure 2). No such definitive pattern of increase was observed for any of the four WD40 repeat proteins investigated. This indicates the regulatory complex of the strawberry is dominated by R2R3-MYB TF, and FaMYB10 with little influence of $b H L H$ counterparts and that the regulatory complex in our studied materials lacks WD40 protein. Orthologs of MYB10 were identified to be involved in the biosynthesis of anthocyanins during ripening of more than 20 different fruits of Rosaceae [25,29]. In strawberry, this gene was found to be expressed in fruit receptacles particularly during ripened to senescent stages compared to earlier stages of fruit development, with negligible expressions being observed in all vegetative tissues (e.g., roots, leaves, crowns, and runners) and in fruit achenes [37].

The composition of the members of this $M B W$ complex is known to vary across species $[3,16,29]$. Among the $M B W$ complex proteins, $b H L H$ and WD proteins have broader and overlapping regulatory targets, while the regulatory targets of $M Y B$ s are known to be specific, as evident by the involvement of different $M Y B$ s for different biosynthetic steps $[3,12,16]$. For apple and grape, only MYB and $b H L H$ proteins (lacking WD40) regulate the biosynthesis of anthocyanin [24,25]. In Arabidopsis, only an independent R2R3-MYB TF lacking both $b H L H$ and WD40 counterparts regulates the early biosynthetic 
genes while the late biosynthetic genes are regulated by $M B W$ complex proteins [16]. The role of $b H L H$ was extensively investigated in rosaceous species and it was observed that the R2R3 domain of MYB10s of 20 rosaceous contains several key motifs that indicates its association with suitable $b H L H$ counterpart [29]. Many of the rosaceous MYBs including strawberry, apple and cherry were observed to promote $D F R$ activity when transiently co-infiltrated with either apple or Arabidopsis $b H L H$ genes. In both wild and cultivated strawberry, the Arabidopsis $b H L H$ genes, AtbHLH2 and AtbHLH42 significantly increased the DFR activity [29]. The promoters of DFR and UFGT of wild strawberry was significantly increased by FvMYB10 and FvbHLH33 as observed by dual luciferase assay in Nicotiana benthamiana [38].

Besides the FaMYB10, significant higher expressions (2.4-2.7 folds) were observed for FaMYB5 in the ripe fruits of high anthocyanin containing cultivars. FaMYB5 also showed significant positive correlation $(p<0.01)$ with FaMYB10 which may indicate the existence of regulatory role of FaMYB5 (Table S1) at least in these high-anthocyanin cultivars. This gene (FaMYB5) was hypothesized to have contrasting positive roles during fruit developmental stages which regulates proanthocyanidin biosynthesis during early- and anthocyanin biosynthesis during late (ripening)-developmental stages [2]. The two bHLH positive regulators, FabHLH3 and FabHLH3-delta, in this study were interestingly found to be the negative regulator of proanthocyanidin biosynthesis in strawberry [2]. The significant and contrastingly higher expressions of FaMYB5, FabHLH3 and FabHLH3-delta, besides $F a M Y B 10$, in the ripe fruits of high anthocyanin containing cultivars and the positive correlation of these genes (which are positively correlated between themselves as well) with total anthocyanin and key structural genes such as FaDFR4-3, FaLDOX and FaUFGT1 indicate their positive contribution in anthocyanin biosynthesis in these cultivars.

\subsection{Potential Repressors of Anthocyanin Biosynthesis}

The contrasting patterns of expressions in high and low anthocyanin cultivars indicate FaMYB11 as potential negative regulator of anthocyanin biosynthesis in the studied genotypes. Besides, expression of FaMYB9 decreased sharply in both high ( $\sim 7$ times) and low ( 15 times) anthocyanin cultivars and the fact that its expression is more decreased in low anthocyanin cultivar indicates its potential role in repressing anthocyanin biosynthesis also (Figure 2). Several negative regulators of anthocyanin biosynthesis is identified in different species such as MdMYB16, MdMYB17 and MdMYB111 in apple [32]; and AtMYB3, AtMYB4 and AtMYBL2 in Arabidopsis [33]. To our best knowledge, no previous report of $F a M Y B 11$ and $F a M Y B 9$ as negative regulator of anthocyanin biosynthesis in Fragaria $\times$ ananassa is available indicating the need for further studies to confirm the exact roles of these genes in strawberry.

FaMYB1 was proposed as a transcriptional repressor of anthocyanin biosynthesis as overexpressing this gene in tobacco has shown to reduce pigmentation via reduced activity of ANS and UFGT genes (of lower end of F/P pathway that leads to the final accumulation of anthocyanin) [30]. However, the gene was found to be highly expressed in red-ripe strawberry fruits [30]. However, its ortholog, FCMYB1, has shown to be highly expressed in white Chilean strawberry (Fragaria chiloensis) compared to that in the red fruits of Fragaria $\times$ ananassa cv. camarosa whose silencing in white Chilean strawberry has increased the level of ANS and reduced those of ANR and LAR reverting the pathway to produce partially red phenotype [31]. In $F$. vesca, the repressor $M Y B 1 R$ was significantly up-regulated in yellow fruits compared to red fruits [39]. We observed differential expression of this genes along green to ripe stages: unaffected in one (Maehyang) and increased in another (Soelhyang) high anthocyanin cultivar, while its expression in the ripe fruits of low anthocyanin cultivar did not change significantly during fruit ripening. This indicates the differential role of this gene in our tested cultivars.

Another $b H L H$ TF, $b H L H 33$, when co-transformed with MYB10, has shown strong activation effect on the apple MYB10 promoter [25] which, however, did not show such activation of the FvMYB10 promoter [38]. However, co-expression of FvbHLH33 with FvMYB10 had shown to strongly activate AtDFR, FvDFR, and FvUFGT promoters in Nicotiana benthamiana plants [38] indicating its positive 
role in activating key anthocyanin pathway genes. We, however, observed a generalized decrease in transcript level of FabHLH33 across fruit developmental stages in all three cultivars. Similar decreasing trend was also observed for FaWD44-1. Thus, besides the contrasting expressions of FaMYB11 in high and low anthocyanin cultivars, which makes it an obvious potential negative regulator, the generalized decreasing trend of expressions of FaMYB9, FabHLH33 and FaWD44-1 along fruit developmental stages in both high and low anthocyanin cultivars also indicates the existence of potential negative influence of these genes on anthocyanin biosynthesis, at least in the studied strawberry cultivars. This is further evident from the fact that these four genes are negatively correlated with the key positive regulator FaMYB10 and with total anthocyanin.

\subsection{Anthocyanin and Proanthocyaninid Might Share Few Contrasting Regulatory Genes}

Discussing the role of regulatory genes of anthocyanin and proanthocyanidin biosynthesis, it became apparent that some regulatory genes may have contrasting roles in these two processes. For example, besides FaMYB10, we observed positive roles of FaMYB5, FabHLH3 and FabHLH3-delta in the biosynthesis of anthocyanin in our strawberry genotypes (Figure 2). Among these, FaMYB5 and FabHLH3-delta were reported as negative regulators of proanthocyanidin biosynthesis in strawberry [2]. While FaMYB11 and FaMYB9 appeared as potential negative regulators of anthocyanin biosynthesis in our study, these two genes were found to act as positive regulator of proanthocyanidin biosynthesis [2]. FaMYB9/FaMYB11 along with FabHLH3 and FaTTG1 form a ternary complex which is shown to upregulate leucoanthocyanidin- and anthocyanidin-reductase ( $L A R$ and $A N R$, respectively) causing an increase in proanthocyanidin contents [2]. Silencing of one of these PA biosynthesis enzymes, ANR has shown to revert the F/P pathway to produce anthocyanin instead of PAs during early developmental stages of strawberry fruits [40]. Contrastingly, overexpression of the Arabidopsis orthologs of these genes (i.e., AtTT2, AtTT8 and AtTTG1, respectively) has shown to decrease anthocyanin and increase PA in strawberry further indicating their contrasting roles in the biosynthesis of two final steps (i.e., biosynthesis of anthocyanins and proanthocyanidins) of F/P pathway. Besides, few genes have shown common effect on accumulation of both anthocyanin and proanthocyanidin such as FabHLH3 and FaTTG1 as evident by their positive roles in PA biosynthesis [2] and higher expression in our high anthocyanin containing ripe fruits.

\subsection{Key Structural Genes and Their Association with Total Anthocyanin and Regulatory Genes}

Based on our comparative univariate expression analysis considering the expressions of green fruits of low anthocyanin cultivar, Tokun as control, we identified FaPAL2, FaCC1, Fa4CL7, FaCHI1, $\mathrm{FaCHI3}, \mathrm{FaCHS}$ and FaF3H as the key early- and FaDFR4-3, FaLDOX and FaUFGT1 as the key late-biosynthetic genes (Figures 2-4). We employed multivariate analytical approach on the entire datasets to identify and visualize the overall association of these genes with total anthocyanins which may have arisen from the patterns of changes in the expressions of the genes and the contents of total anthocyanin in the three progressive fruit developmental stages of high- and low-anthocyanin cultivars. Positioning of the highly expressed genes along with total anthocyanin content and high anthocyanin containing ripe fruit samples of Maehyang and Soelhyang in close vicinity in PCA biplot indicates the close association between these factors which can be translated to the fact that the activity of these genes in ripe fruits of Maehyang and Soelhyang leads to the higher accumulation of anthocyanin. With total anthocyanin and FaMYB10, the key regulator, only these structural genes have shown higher significant positive correlations which further corroborates the association between these genes and total anthocyanin. A previous study, however, reported no determining role of $4 \mathrm{CL}$ in strawberry fruit pigmentation [31], whereas we observed 17.8- and 16.2-fold higher expression of this gene in the ripe fruits of our high anthocyanin containing cultivars compared to the respective green fruits.

The relationship between the key regulatory gene, FaMYB10 with other structural genes was previously demonstrated by several transcriptomic, over-expression and gene silencing studies [7,10,37-39]. For example, over-expression of FaMYB10 had increased anthocyanin contents in 
roots, leaves and fruits of Fragaria $\times$ ananassa [29] and silencing of this genes had downregulated both early- and late-biosynthetic genes of F/P pathway that includes PAL, C4H, F3H, 4CL, CHS, CHI, DFR and UFGT, etc. [37]. A similar set of structural genes were reported in several other species as well such as in grapevine [17], pear [41], apple [42], potato tuber [43], etc. A little contrast is observed in our correlation based analysis as the gene $\mathrm{FaC} 4 \mathrm{H}$ was found to be negatively correlated (statistically non-significant) with both total anthocyanin and FaMYB10 (Table S1). Furthermore, silencing of FaMYB10 (and also FvMYB10) did not show any significant effect on the expression of LDOX (or ANS) [38]; which prompted to speculate different regulatory mechanism for this gene manifested by another MYB transcription regulator FaMYB5 (whose expression is not regulated by FaMYB10) [37]. We observed high significant positive correlation of FaLDOX with both FaMYB10 and FaMYB5 (with the latter two showing significant positive correlation between themselves too) which indicates the existence of regulatory roles of these two TFs. It is notable to mention in this regard that besides FaLDOX; FaMYB5 also showed significant positive correlations with all the key structural genes to which FaMYB10 too showed positive correlation (Table S1). Our results with FaMYB10 seemed to be consistent with the findings that stable over-expression of its F. vesca counterpart, FvMYB10 had increased the expression of $L D O X$ [38] as opposed to the previous speculation of having different roles in regulating $L D O X$ transcription. As with the specific role of FaMYB5, which was contrastingly found to act as a negative regulator of proanthocyanidin biosynthesis in F. ananassa [2], further functional investigation is necessary.

\subsection{Progressive Intensification of Pathway Flux May Lead to Higher Anthocyanin Accumulation}

Anthocyanin biosynthesis is a complex multi-enzymatic process requiring the coordinated interaction and systemic expressions of many genes via a highly regulated mechanism within the limits of developmental stages and environmental cues that control the pathway flux across the branch points leading to the final accumulation of the end products $[3,5,10,12,16]$. A combined study of anthocyanin biosynthesis focusing on the genetic, developmental and environmental influences revealed that expressions of genes, activities of enzymes and levels of flavonoids, all follow a clear developmental pattern in strawberry [14]. An overview on the highly expressed genes of each of the pathway nodes in our study makes it apparent that late biosynthetic genes are comparatively highly expressed compared to the early biosynthetic genes and a general increasing trend of gene expressions (starting from FaPAL1 having a maximum of 2.14-fold expression through to FaUFGT2 having a maximum of $~ 384$-fold expression, with $\mathrm{FaCHS}$ and $\mathrm{FaF} 3 \mathrm{H}$ causing little fluctuation in this trend) is observed along the progress of the pathway which probably indicates the progressive intensification of the metabolic flux leading to the final accumulation of anthocyanin (Figure S1). Early biosynthetic genes (such as CHS, $\mathrm{CHI}, \mathrm{F3H}$, etc.) are known to catalyze the production of flavonols whereas the late biosynthetic genes (DFR, LDOX/ANS and UFGTs) are involved in biosynthesis of anthocyanin [44,45]. The existence of different sets of regulatory gene(s) as proposed for the early and late biosynthetic steps [44] may have a role in this differential expression of the early- and late-biosynthetic genes.

\section{Methods}

\subsection{Plant Materials}

Three strawberry (Fragaria $\times$ ananassa) cultivars, namely, Maehyang, Seolhyang and Tokun (also known as "Toukun") were grown in large rectangular pots using nursery soil mix under standard growth and nutritional conditions in the glass house research facility of Suncheon National University, South Korea. Fruits of three developmental stages, namely, green, white and ripe stages were harvested and were immediately flash frozen in liquid nitrogen before storing at $-80{ }^{\circ} \mathrm{C}$ for the subsequent quantification of total anthocyanin and extraction of total RNA. 


\subsection{Extraction and Photometric Determination of Anthocyanin}

Total anthocyanins were extracted from the liquid nitrogen frozen fruit samples following the procedures described by [35] with minor modifications. In short, anthocyanin were extracted in $1 \mathrm{~mL}$ of acidic methanol $(1 \% \mathrm{HCl}, w / v)$ by incubating $100 \mathrm{mg}$ of finely grounded fruit tissue powder at room temperature for $18 \mathrm{~h}$ in dark followed by clearing up the extract by centrifugation at 14,000 rpm for $10 \mathrm{~min}$. Total anthocyanins were quantified based on the absorption of the extracts using the equation: $Q_{\text {Anthocyanins }}=\left(A_{530}-0.25 \times A_{657}\right) \times F W^{-1}$, where $Q_{\text {Anthocyanins }}$ is the amount of total anthocyanins; $A_{530}$ and $A_{657}$ are the absorptions at $530 \mathrm{~nm}$ and $657 \mathrm{~nm}$, respectively; and $F W$ is the weight of plant materials $(\mathrm{g})$. Anthocyanins were quantified as triplicates of three independent biological replicates.

\subsection{Selection of Anthocyanin Related Genes in Fragaria $\times$ ananassa}

The genes involved in each step of the anthocyanin biosynthesis pathway were first manually searched using step-specific key words (such as "DFR", "Dihydroflavanol", etc. for identifying the DFR genes) from the annotated version of the whole genome of wild strawberry, Fragaria vesca ("Fvesca_V1.0_genemark_hybrid annotation" file, available from the Genome Database of Rosaceae in https: / / www.rosaceae.org/) (Table S2). Among these genes, the important regulatory, early- and late-biosynthetic genes whose expressions were to be studied in cultivated strawberry, Fragaria $\times$ ananassa were then selected based on their prior reports in previous studies on Fragaria vesca and/or Fragaria $\times$ ananassa. The F. vesca (wild strawberry) sequences of selected genes (from Table S2) were then used to identify corresponding Fragaria $\times$ ananassa (cultivated strawberry) sequences (including the isoforms) by using the "BLAST" tool against the Fragaria $\times$ ananassa draft genome (FANhybrid_r1.2_cds, available from the Strawberry garden database-http:/ / strawberry-garden. kazusa.or.jp/). The list of the selected genes is shown in Table 2; the stepwise biosynthetic genes are marked in anthocyanin biosynthesis pathway in Figure 6; and the complete information are given in Table S3.

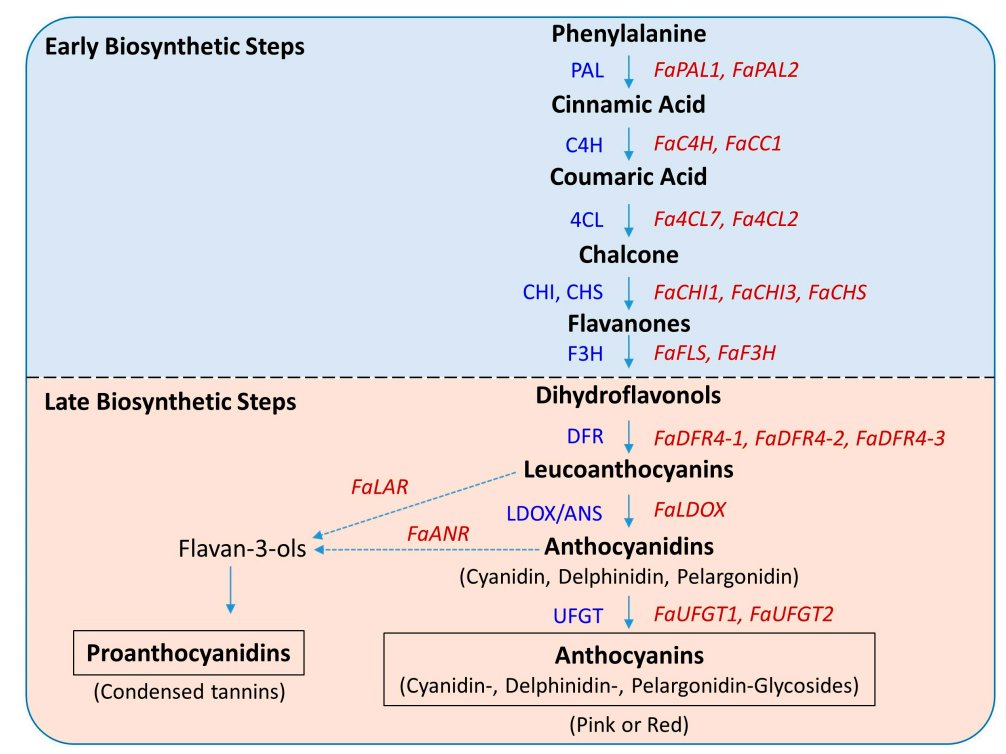

Figure 6. Simplified schematic representation of phenylapropanoid/flavonoid biosynthesis pathway leading to the accumulation of anthocyanins in Fragaria $\times$ ananassa. Enzymes involved in each of the intermediate steps are shown by blue text in the left side of the arrow and the genes whose expressions were studied were shown by red text in the right side of the arrow. Key flavonoid end products are shown in boxes. Dotted arrows indicate the points where the pathway diverts from biosynthesis of anthocyanin to proanthocyanidin. Details of the genes involved in each of the steps are shown in Tables 2 and S3. 
Table 2. List of the genes investigated for their involvement in the regulation and early- and late-biosynthesis of anthocyanin in Fragaria $\times$ ananassa. The genes were first manually mined from F. vesca (Fvesca_V1.0_genemark_hybrid annotation) genome (Table S2) and then the corresponding sequences of the selected genes were obtained by blasting against Fragaria $\times$ ananassa genome (FANhybrid_r1.2_cds) available from the Strawberry garden database (http://strawberry-garden.kazusa.or.jp/).

\begin{tabular}{|c|c|c|c|c|c|}
\hline Gene & $\begin{array}{l}\text { Gene ID in Fragaria } \times \text { ananassa } \\
\text { (FANhybrid_r1.2_cds) }\end{array}$ & $\begin{array}{l}\text { CDS } \\
(\text { bp) }\end{array}$ & Annotation & $\begin{array}{l}\text { Best Match of } F \\
\text { vesca Gene }\end{array}$ & $e$-Value \\
\hline \multicolumn{6}{|c|}{ Regulatory Genes } \\
\hline \multicolumn{6}{|l|}{$M Y B$} \\
\hline FaMYB11 & FANhyb_icon00023020_a.1.g00001.1/partial & 425 & MYB11.m1; organism $=$ Fragaria $\times$ ananassa & gene07416 & 0.00 \\
\hline FaMYB1 & FANhyb_icon00014430_a.1.g00001.1/partial & 272 & MYB1.m1; organism $=$ Fragaria $\times$ ananassa & gene09407 & $1 \times 10^{-153}$ \\
\hline FaMYB10 & FANhyb_icon00002569_a.1.g00001.1/partial & 233 & Transcription factor MYB113 (AtMYB113) (simiLAR to) & gene31413 & $1 \times 10^{-119}$ \\
\hline FaMYB5 & FANhyb_rscf00000101.1.g00008.1 & 1071 & MYB5.m1; organism $=$ Fragaria $\times$ ananassa & gene24821 & 0.00 \\
\hline FаMҮB9 & FANhyb_rscf00002302.1.g00001.1/partial & 828 & MYB9.m1; organism $=$ Fragaria $\times$ ananassa & gene15392 & 0.00 \\
\hline FaEOBII & FANhyb_rscf00000047.1.g00023.1/TE & 393 & MYB-related protein 305 (putative) & gene28435 & 0.00 \\
\hline \multicolumn{6}{|l|}{ bHLH } \\
\hline FabHLH33 & FANhyb_rscf00000043.1.g00015.1 & 1962 & BHLH33.m1; organism $=$ Fragaria $\times$ ananassa & gene19321 & 0.00 \\
\hline FabHLH3-delta & FANhyb_icon00003421_a.1.g00001.1/partial & 557 & Transcription factor TT8 (bHLH 42) (simiLAR to) & gene27827 & 0.00 \\
\hline FabHLH3 & FANhyb_rscf00003752.1.g00002.1 & 1041 & Transcription factor TT8 ( $b H L H 42)$ (simiLAR to) & gene27827 & 0.00 \\
\hline FabHLH1 & FANhyb_icon00000044_a.1.g00001.1 & 1999 & Transcription factor GLABRA 3 (bHLH 1) (putative); FaMYC1 mRNA & gene32494 & 0.00 \\
\hline \multicolumn{6}{|l|}{ WD40 } \\
\hline FaWD40-1 & FANhyb_rscf00000569.1.g00002.1 & 1539 & WD40 repeat-containing protein SMU1 (putative) & gene27104 & 0.00 \\
\hline FaWD-1 & FANhyb_icon00020056_a.1.g00001.1/partial & 152 & WD repeat-containing protein mio (probable) & gene03735 & $3 \times 10^{-71}$ \\
\hline FaTTG1 & FANhyb_icon00009619_a.1.g00001.1/partial & 367 & Protein transparent Testa Glabra 1 (simiLAR to); TTG1 & gene12450 & 0.00 \\
\hline FAWD44-1 & FANhyb_rscf00002089.1.g00001.1 & 2067 & Fragaria vesca WD repeat-containing protein 44 & gene17869 & 0.00 \\
\hline \multicolumn{6}{|c|}{ Early Biosynthetic Genes } \\
\hline \multicolumn{6}{|l|}{ Phenylalanine } \\
\hline FaPAL1 & FANhyb_rscf00000868.1.g00006.1 & 1674 & Phenylalanine ammonia-lyase 1 & gene23261 & 0.00 \\
\hline FaPAL2 & FANhyb_rscf00000079.1.g00001.1 & 2175 & Phenylalanine ammonia-lyase 2 (putative) & gene09753 & 0.00 \\
\hline \multicolumn{6}{|l|}{ Cinnamic Acid } \\
\hline $\mathrm{FaC} 4 \mathrm{H}$ & FANhyb_rscf00000282.1.g00007.1 & 789 & Trans-cinnamate 4-monooxygenase (CA4H) (putative) & gene28093 & 0.00 \\
\hline $\mathrm{FaCC1}$ & FANhyb_rscf00000685.1.g00004.1 & 6963 & Biotin carboxylase (probable); Acetyl-CoA carboxylase, AtACC1 & gene22077 & 0.00 \\
\hline
\end{tabular}


Table 2. Cont.

\begin{tabular}{|c|c|c|c|c|c|}
\hline Gene & $\begin{array}{l}\text { Gene ID in Fragaria } \times \text { ananassa } \\
\text { (FANhybrid_r1.2_cds) }\end{array}$ & $\begin{array}{l}\text { CDS } \\
\text { (bp) }\end{array}$ & Annotation & $\begin{array}{l}\text { Best Match of } F \\
\quad \text { vesca Gene }\end{array}$ & $e$-Value \\
\hline \multicolumn{6}{|c|}{ Coumaroyl-CoA } \\
\hline Fa4CL7 & FANhyb_icon00012602_a.1.g00001.1/partial & 352 & 4-coumarate-CoA ligase-like 7 (At4CL6) (putative) & gene09603 & 0.00 \\
\hline Fa4CL2 & FANhyb_rscf00001339.1.g00001.1 & 1644 & 4-coumarate-CoA ligase 2 (4CL 2) (putative) & gene15877 & 0.00 \\
\hline \multicolumn{6}{|l|}{ Chalcone } \\
\hline $\mathrm{FaCHI3}$ & FANhyb_icon00000880_a.1.g00001.1/partial & 638 & Chalcone-flavonone isomerase (probable) & gene21346 & 0.00 \\
\hline $\mathrm{FaCHI} 1$ & FANhyb_icon00004487_a.1.g00001.1/partial & 534 & Chalcone-flavonone isomerase 1 ; TT5 & gene23367 & 0.00 \\
\hline $\mathrm{FaCHS}$ & FANhyb_icon00003433_a.1.g00001.1/partial & 992 & Chalcone synthase, TT4; FvCHS & gene26825 & 0.00 \\
\hline \multicolumn{6}{|l|}{ Flavanone } \\
\hline FaFLS & FANhyb_icon00020196_a.1.g00001.1/partial & 242 & Flavonol synthase/flavanone 3-hydroxylase (FLS) (simiLAR to) & gene11126 & $1 \times 10^{-111}$ \\
\hline $\mathrm{FaF3H}$ & FANhyb_icon00001777_a.1.g00001.1 & 825 & Naringenin,2-oxoglutarate 3-dioxygenase (F3H) (putative) & gene14611 & 0.00 \\
\hline \multicolumn{6}{|c|}{ Late Biosynthetic Genes } \\
\hline \multicolumn{6}{|c|}{ Dihydroflavonol } \\
\hline FaDFR4-1 & FANhyb_rscf00006583.1.g00001.1/partial & 578 & Bifunctional dihydroflavonol 4-reductase (DFR) (probable) & gene29482 & $1 \times 10^{-143}$ \\
\hline FaDFR4-2 & FANhyb_rscf00000482.1.g00004.1 & 2031 & Bifunctional dihydroflavonol 4-reductase (DFR) (putative) & gene15174 & 0.00 \\
\hline FaDFR4-3 & FANhyb_rscf00000482.1.g00003.1 & 1050 & Bifunctional dihydroflavonol 4-reductase (DFR) (simiLAR to) & gene15176 & 0.00 \\
\hline \multicolumn{6}{|c|}{ Leucoanthocyanidin } \\
\hline FaLDOX & FANhyb_icon00007826_a.1.g00001.1/partial & 794 & Leucoanthocyanidin dioxygenase (LDOX) (putative); FvLDOX, TT18 & gene32347 & 0.00 \\
\hline FaANR & FANhyb_rscf00000390.1.g00012.1/partial & 887 & Leucoanthocyanidin reductase (LAR) (putative) & gene24665 & 0.00 \\
\hline FaLAR & FANhyb_icon00014507_a.1.g00001.1/partial & 497 & Leucoanthocyanidin reductase (simiLAR to) & gene03877 & 0.00 \\
\hline \multicolumn{6}{|c|}{ Anthocyanidin } \\
\hline FaUFGT1 & FANhyb_rscf00000061.1.g00006.1 & 1347 & Anthocyanidin 3-O-glucosyltransferase 2 (putative) & gene12591 & 0.00 \\
\hline FaUFGT2 & FANhyb_rscf00000877.1.g00006.1 & 1299 & Anthocyanidin $5,3-O$-glucosyltransferase (probable) & gene04355 & 0.00 \\
\hline
\end{tabular}




\subsection{RNA Extraction and Expression Analysis via Real-Time $q R T-P C R$}

Total RNA was isolated from the liquid nitrogen frozen fruit samples of three fruit developmental stages, namely, green, white and ripening stages, using RNeasy mini kit (Qiagen, Inc., Redwood, CA, USA) based on manufacturer's instruction. The samples were treated with RNAse free DNase (Qiagen) to remove any genomic DNA contamination. Conversion of total RNA into cDNA was carried out using Superscript-III ${ }^{\circledR}$ First-strand Synthesis Supermix kit (Invitrogen, Carlsbad, CA, USA). The purity and concentration were determined spectrophotometrically using Nanodrop-2000 (Nanodrop Technologies, Wilmington, DE, USA).

The qRT-PCR based expression profiling of the genes were carried out using "Roche-Light Cycler ${ }^{\circledR}$ 96 system" (Roche Diagnostics, Pleasanton, CA, USA). The gene-specific primers were designed for each of the genes using primer3plus (http://primer3plus.com/cgi-bin/dev/primer3plus.cgi) (Table S4). For each reverse transcription reaction, a total volume of $20 \mu \mathrm{L}$ was prepared containing $10 \mu \mathrm{L}$ of $2 \times$ qPCRBIO SyGreen Mix (PCR Biosystems, London, UK), $1 \mu \mathrm{L}$ of each of the forward and reverse primers (10 pmoles) and $60 \mathrm{ng} / \mu \mathrm{L}$ of cDNA as template. The qRT-PCR was carried out with denaturation at $95{ }^{\circ} \mathrm{C}$ for $10 \mathrm{~min}$, and 45 cycles of amplification with denaturation at $95{ }^{\circ} \mathrm{C}(20 \mathrm{~s})$, annealing at $55^{\circ} \mathrm{C}(20 \mathrm{~s})$ and elongation at $72{ }^{\circ} \mathrm{C}(25 \mathrm{~s})$. Each of the three biological replicates were tested in three technical replicates. Primer specificity was checked by single melting peak. Relative expression for each gene was quantified following Livak's comparative $2^{-\Delta \Delta C t}$ method using a Light Cycler $^{\circledR} 96$ Instrument (Roche Diagnostics, Indianapolis, IN, USA.) [46]. FaRIB413 was used as the reference gene for expression analysis [47]. Relative expressions for each of the genes were calculated relative to the expression of the respective genes in the green fruit of cultivar, Tokun (control) which was assigned an arbitrary value equal to unity.

\subsection{Statistical Analysis}

Total anthocyanin contents and gene expressions were analyzed by one-way ANOVA and statistically significant differences were analyzed by Tukey's pairwise comparisons. Data are presented as the average of 3 replicates with error bar indicating standard deviation. The data were standardized (mean subtracted from the variable and then divided by the standard deviation) prior to perform Principal component analysis (PCA). The correlation between the genes and total anthocyanin were measured by Pearson correlation analysis and tested for statistical significance. All statistical analysis was conducted using Minitab v. 17 statistical packages (Minitab Inc., State College, PA, USA).

\section{Conclusions}

This study identified the potential regulators involved in the biosynthesis of anthocyanins in contrastingly anthocyanin rich strawberry cultivars. Multivariate statistics based analytical approaches helped to gain a holistic scenario of overall associations of the genetic determinants involved in the entire process. The results are discussed within the wider context of existing body of knowledge that lead to the identification of few genes having important and/or deferential roles besides the previously known genes. Functional validation of these genes will further widen our understanding of the mechanisms involved. Identification of the key genes will thus be helpful in channeling future efforts towards developing better varieties with improved anthocyanin related traits via breeding and biotechnological means.

Supplementary Materials: Supplementary Materials can be found at www.mdpi.com/1422-0067/19/3/656/s1.

Acknowledgments: This study was supported by the Golden Seed Project (Center for Horticultural Seed Development, Grant No. 213007-05-2-CG100 and Strawberry Project, Grant No. 315049-03-3-HD050) of the Ministry of Agriculture, Food and Rural affairs in the Republic of Korea (MAFRA).

Author Contributions: Ill-Sup Nou, Jong-In Park, Hoy-Taek Kim and Mohammad Rashed Hossain conceived and designed the study. Mohammad Rashed Hossain conducted the experiments, did the statistical analysis and wrote the manuscript. Ashokraj Shanmugam assisted in sample collection, RNA extraction and cDNA synthesis. 
Ujjal Kumar Nath and Gayatri Goswami assisted in qPCR analysis. Jae-Young Song provided the plant materials and assisted in editing.

Conflicts of Interest: The authors declare no conflicts of interest.

$\begin{array}{ll}\text { Abbreviations } \\ \text { F/P } & \text { Phenylapropanoid/flavonoid pathway } \\ \text { BGs } & \text { Biosynthetic Genes } \\ \text { EBGs } & \text { Early Biosynthetic Genes } \\ \text { LBGs } & \text { Late Biosynthetic Genes } \\ \text { TF } & \text { Transcription Factor } \\ \text { MYB } & \text { Myeloblastosis family of transcription factors } \\ \text { bHLH } & \text { Basic helix-loop-helix } \\ \text { PAL } & \text { Phenylalanine ammonia lyase } \\ \text { C4H } & \text { Cinnamate-4-hydroxylase } \\ \text { 4CL } & \text { 4-Coumaroyl-CoA-ligase } \\ \text { CHS } & \text { Chalcone synthase } \\ \text { CHI } & \text { Chalcone isomerase } \\ \text { F3H } & \text { Flavanone 3 hydroxylase } \\ \text { FLS } & \text { Flavonol synthase } \\ \text { DFR } & \text { Dihydroflavanol reductase } \\ \text { LAR } & \text { Leucoanthocyanidin reductase } \\ \text { ANR } & \text { Anthocyanidin reductase }\end{array}$

\section{References}

1. Mezzetti, B. Breeding and biotechnology for improving the nutritional quality of strawberry. J. Berry Res. 2013, 3, 127-133.

2. Schaart, J.G.; Dubos, C.; Romero De La Fuente, I.; van Houwelingen, A.M.M.L.; de Vos, R.C.H.; Jonker, H.H.; Xu, W.; Routaboul, J.M.; Lepiniec, L.; Bovy, A.G. Identification and characterization of MYB-bHLH-WD40 regulatory complexes controlling proanthocyanidin biosynthesis in strawberry (Fragaria $\times$ ananassa) fruits. New Phytol. 2013, 197, 454-467. [CrossRef] [PubMed]

3. Jaakola, L. New insights into the regulation of anthocyanin biosynthesis in fruits. Trends Plant Sci. 2013, 18, 477-483. [CrossRef] [PubMed]

4. Kayesh, E.; Shangguan, L.; Korir, N.K.; Sun, X.; Bilkish, N.; Zhang, Y.; Han, J.; Song, C.; Cheng, Z.M.; Fang, J. Fruit skin color and the role of anthocyanin. Acta Physiol. Plant. 2013, 35, 2879-2890. [CrossRef]

5. Tanaka, Y.; Sasaki, N.; Ohmiya, A. Biosynthesis of plant pigments: Anthocyanins, betalains and carotenoids. Plant J. 2008, 54, 733-749. [CrossRef] [PubMed]

6. Jimenez-Garcia, S.N.; Guevara-Gonzalez, R.G.; Miranda-Lopez, R.; Feregrino-Perez, A.A.; Torres-Pacheco, I.; Vazquez-Cruz, M.A. Functional properties and quality characteristics of bioactive compounds in berries: Biochemistry, biotechnology, and genomics. Food Res. Int. 2013, 54, 1195-1207. [CrossRef]

7. Lin-Wang, K.; Liu, Y.; Espley, R.V.; Karunairetnam, S.; McGhie, T.K.; Hellens, R.P.; Allan, A.C. Regulation of anthocyanin biosynthesis in strawberry (Fragaria sp.) by over-expression of a key transcription factor. Acta Hortic. 2014, 1048, 137-142. [CrossRef]

8. Schaefer, H.M.; Schaefer, V.; Levey, D.J. How plant-animal interactions signal new insights in communication. Trends Ecol. Evol. 2004, 19, 577-584. [CrossRef]

9. Park, K.-I.; Ishikawa, N.; Morita, Y.; Choi, J.-D.; Hoshino, A.; Iida, S. A bHLH regulatory gene in the common morning glory, Ipomoea purpurea, controls anthocyanin biosynthesis in flowers, proanthocyanidin and phytomelanin pigmentation in seeds, and seed trichome formation. Plant J. 2007, 49, 641-654. [CrossRef] [PubMed] 
10. Pillet, J.; Yu, H.; Chambers, A.H.; Whitaker, V.M.; Folta, K.M. Identification of candidate flavonoid pathway genes using transcriptome correlation network analysis in ripe strawberry (Fragaria $\times$ ananassa) fruits. J. Exp. Bot. 2015, 66, 4455-4467. [CrossRef] [PubMed]

11. Koide, T.; Kamei, H.; Hashimoto, Y.; Kojima, T.; Hasegawa, M. Antitumor effect of hydrolyzed anthocyanin from grape rinds and red rice. Cancer Biother. Radiopharm. 1996, 11, 273-277. [CrossRef] [PubMed]

12. Cristina, L.; Aizza, B.; Dornelas, M.C. Differential Transcription Factor Networks Orchestrate Flavonoid Biosynthesis. In Pigments in Fruits and Vegetables; Chen, C., Ed.; Springer: New York, NY, 2015; pp. 69-91.

13. Almeida, J.R.M.; D'Amico, E.; Preuss, A.; Carbone, F.; Deiml, B.; Mourgues, F.; Perrotta, G.; Fischer, T.C.; Bovy, A.G.; Martens, S.; Rosati, C. Characterization of major enzymes and genes involved in flavonoid and proanthocyanidin biosynthesis during fruit development in strawberry (Fragaria $\times$ ananassa). Arch. Biochem. Biophys. 2007, 465, 61-71. [CrossRef] [PubMed]

14. Carbone, F.; Preuss, A.; De Vos, R.C.; Amico, E.D.; Perrotta, G.; Bovy, A.G.; Martens, S.; Rosati, C. Developmental, genetic and environmental factors affect the expression of flavonoid genes, enzymes and metabolites in strawberry fruits. Plant Cell Environ. 2009, 32, 1117-1131. [CrossRef] [PubMed]

15. Afrin, S.; Nuruzzaman, M.; Zhu, J.; Luo, Z. Combinatorial interactions of MYB and $b H L H$ in flavonoid biosynthesis and their function in plants. J. Plant Biol. Res. 2014, 3, 65-77.

16. Petroni, K.; Tonelli, C. Recent advances on the regulation of anthocyanin synthesis in reproductive organs. Plant Sci. 2011, 181, 219-229. [CrossRef] [PubMed]

17. Hichri, I.; Barrieu, F.; Bogs, J.; Kappel, C.; Delrot, S.; Lauvergeat, V. Recent advances in the transcriptional regulation of the flavonoid biosynthetic pathway. J. Exp. Bot. 2011, 62, 2465-2483. [CrossRef] [PubMed]

18. Allan, A.C.; Hellens, R.P.; Laing, W.A. MYB transcription factors that colour our fruit. Trends Plant Sci. 2008, 13, 99-102. [CrossRef] [PubMed]

19. Gonzalez, A.; Zhao, M.; Leavitt, J.M.; Lloyd, A.M. Regulation of the anthocyanin biosynthetic pathway by the TTG1/bHLH/MYB transcriptional complex in Arabidopsis seedlings. Plant J. 2008, 53, 814-827. [CrossRef] [PubMed]

20. Cutanda-Perez, M.-C.; Ageorges, A.; Gomez, C.; Vialet, S.; Terrier, N.; Romieu, C.; Torregrosa, L. Ectopic expression of VIMYBA1 in grapevine activates a narrow set of genes involved in anthocyanin synthesis and transport. Plant Mol. Biol. 2009, 69, 633-648. [CrossRef] [PubMed]

21. Borovsky, Y.; Oren-Shamir, M.; Ovadia, R.; De Jong, W.; Paran, I. The A locus that controls anthocyanin accumulation in pepper encodes a $M Y B$ transcription factor homologous to Anthocyanin2 of Petunia. Theor. Appl. Genet. 2004, 109, 23-29. [CrossRef] [PubMed]

22. Grotewold, E.; Sainz, M.B.; Tagliani, L.; Hernandez, J.M.; Bowen, B.; Chandler, V.L. Identification of the residues in the MYB domain of maize $\mathrm{C} 1$ that specify the interaction with the bHLH cofactor R. Proc. Natl. Acad. Sci. USA 2000, 97, 13579-13584. [CrossRef] [PubMed]

23. Liu, Y.; Lin-Wang, K.; Espley, R.V.; Wang, L.; Yang, H.; Yu, B.; Dare, A.; Varkonyi-Gasic, E.; Wang, J.; Zhang, J.; et al. Functional diversification of the potato R2R3 MYB anthocyanin activators AN1, MYBA1, and MYB113 and their interaction with basic helix-loop-helix cofactors. J. Exp. Bot. 2016, 67, 2159-2176. [CrossRef] [PubMed]

24. Ban, Y.; Honda, C.; Hatsuyama, Y.; Igarashi, M.; Bessho, H.; MoriguCHI, T. Isolation and Functional Analysis of a MYB Transcription Factor Gene that is a Key Regulator for the Development of Red Coloration in Apple Skin. Plant Cell Physiol. 2007, 48, 958-970. [CrossRef] [PubMed]

25. Espley, R.V.; Hellens, R.P.; Putterill, J.; Stevenson, D.E.; Kutty-Amma, S.; Allan, A.C. Red colouration in apple fruit is due to the activity of the MYB transcription factor, MdMYB10. Plant J. 2007, 49, 414-427. [CrossRef] [PubMed]

26. Jin, W.; Wang, H.; Li, M.; Wang, J.; Yang, Y.; Zhang, X.; Yan, G.; Zhang, H.; Liu, J.; Zhang, K. The R2R3 MYB transcription factor PavMYB10.1 involves in anthocyanin biosynthesis and determines fruit skin colour in sweet cherry (Prunus avium L.). Plant Biotechnol. J. 2016, 14, 2120-2133. [CrossRef] [PubMed]

27. Wei, H.; Chen, X.; Zong, X.; Shu, H.; Gao, D. Comparative Transcriptome Analysis of Genes Involved in Anthocyanin Biosynthesis in the Red and Yellow Fruits of Sweet Cherry (Prunus avium L.). PLoS ONE 2015, 10, 1-20. [CrossRef] [PubMed] 
28. Carey, C.C.; Strahle, J.T.; Selinger, D.A.; Chandler, V.L. Mutations in the pale aleurone color1 Regulatory Gene of the Zea mays Anthocyanin Pathway Have Distinct Phenotypes Relative to the Functionally SimiLAR TRANSPARENT TESTA GLABRA1 Gene in Arabidopsis thaliana. Plant Cell 2004, 16, 450-464. [CrossRef] [PubMed]

29. Lin-wang, K.; Bolitho, K.; Grafton, K.; Kortstee, A.; Karunairetnam, S.; Mcghie, T.K.; Espley, R.V.; Hellens, R.P.; Allan, A.C. An R2R3 MYB transcription factor associated with regulation of the anthocyanin biosynthetic pathway in Rosaceae. BMC Plant Biol. 2010, 10, 50. [CrossRef] [PubMed]

30. Aharoni, A.; De Vos, C.H.R.; Wein, M.; Sun, Z.; Greco, R.; Kroon, A.; Mol, J.N.M.; O'Connell, A.P. The strawberry FaMYB1 transcription factor suppresses anthocyanin and flavonol accumulation in transgenic tobacco. Plant J. 2001, 28, 319-332. [CrossRef] [PubMed]

31. Salvatierra, A.; Pimentel, P.; Moya-León, M.A.; Herrera, R. Increased accumulation of anthocyanins in Fragaria chiloensis fruits by transient suppression of FcMYB1 gene. Phytochemistry 2013, 90, 25-36. [CrossRef] [PubMed]

32. Lin-Wang, K.; Micheletti, D.; Palmer, J.; Volz, R.; Lozano, L.; Espley, R.; Hellens, R.P.; Chagne, D.; Rowan, D.D.; Troggio, M.; et al. High temperature reduces apple fruit colour via modulation of the anthocyanin regulatory complex. Plant. Cell Environ. 2011, 34, 1176-1190. [CrossRef] [PubMed]

33. Dubos, C.; Le Gourrierec, J.; Baudry, A.; Huep, G.; Lanet, E.; Debeaujon, I.; Routaboul, J.-M.; Alboresi, A.; Weisshaar, B.; Lepiniec, L. MYBL2 is a new regulator of flavonoid biosynthesis in Arabidopsis thaliana. Plant J. 2008, 55, 940-953. [CrossRef] [PubMed]

34. Miao, L.; Zhang, Y.; Yang, X.; Xiao, J.; Zhang, H.; Zhang, Z.; Wang, Y.; Jiang, G. Colored light-quality selective plastic films affect anthocyanin content, enzyme activities, and the expression of flavonoid genes in strawberry (Fragaria $\times$ ananassa) fruit. Food Chem. 2016, 207, 93-100. [CrossRef] [PubMed]

35. Rabino, I.; Mancinelli, A.L. Light, temperature, and anthocyanin production. Plant Physiol. 1986, 81, $922-924$. [CrossRef] [PubMed]

36. Takos, A.M.; Jaffe, F.W.; Jacob, S.R.; Bogs, J.; Robinson, S.P.; Walker, A.R. Light-Induced Expression of a MYB Gene Regulates Anthocyanin Biosynthesis in Red Apples. Plant Physiol. 2006, 142, 1216-1232. [CrossRef] [PubMed]

37. Medina-Puche, L.; Cumplido-Laso, G.; Amil-Ruiz, F.; Hoffmann, T.; Ring, L.; Rodríguez-Franco, A.; Caballero, J.L.; Schwab, W.; Muñoz-Blanco, J.; Blanco-Portales, R. MYB10 plays a major role in the regulation of flavonoid/phenylpropanoid metabolism during ripening of Fragaria $\times$ ananassa fruits. J. Exp. Bot. 2014, 65, 401-417. [CrossRef] [PubMed]

38. Lin-Wang, K.; McGhie, T.K.; Wang, M.; Liu, Y.; Warren, B.; Storey, R.; Espley, R.V.; Allan, A.C. Engineering the anthocyanin regulatory complex of strawberry (Fragaria vesca). Front. Plant Sci. 2014, 5, 1-15. [CrossRef] [PubMed]

39. Zhang, Y.; Li, W.; Dou, Y.; Zhang, J.; Jiang, G.; Miao, L.; Han, G.; Liu, Y.; Li, H.; Zhang, Z. Transcript Quantification by RNA-Seq Reveals Differentially Expressed Genes in the Red and Yellow Fruits of Fragaria vesca. PLoS ONE 2015, 10, 1-15. [CrossRef] [PubMed]

40. Fischer, T.C.; Mirbeth, B.; Rentsch, J.; Sutter, C.; Ring, L.; Flachowsky, H.; Habegger, R.; Hoffmann, T.; Hanke, M.-V.; Schwab, W. Premature and ectopic anthocyanin formation by silencing of anthocyanidin reductase in strawberry (Fragaria $\times$ ananassa). New Phytol. 2014, 201, 440-451. [CrossRef] [PubMed]

41. Zhang, X.; C Allan, A.; Yi, Q.; Chen, L.; Li, K.; Shu, Q.; Su, J. Differential Gene Expression Analysis of Yunnan Red Pear, Pyrus Pyrifolia, During Fruit Skin Coloration. Plant Mol. Biol. Report. 2011, 29, 305-314. [CrossRef]

42. Lister, C.E.; Lancaster, J.E.; Walker, J.R.L. Developmental Changes in Enzymes of Flavonoid Biosynthesis in the Skins of Red and Green Apple Cultivars. J. Sci. Food Agric. 1996, 71, 313-320. [CrossRef]

43. Zhang, H.; Yang, B.; Liu, J.; Guo, D.; Hou, J.; Chen, S. Analysis of structural genes and key transcription factors related to anthocyanin biosynthesis in potato tubers. Sci. Hortic. 2017, 225, 310-316. [CrossRef]

44. Zhang, L.; Xu, B.; Wu, T.; Yang, Y.; Fan, L.; Wen, M.; Sui, J. Transcriptomic profiling of two Pak Choi varieties with contrasting anthocyanin contents provides an insight into structural and regulatory genes in anthocyanin biosynthetic pathway. BMC Genom. 2017, 18, 288. [CrossRef] [PubMed]

45. Guo, N.; Cheng, F.; Wu, J.; Liu, B.; Zheng, S.; Liang, J.; Wang, X. Anthocyanin biosynthetic genes in Brassica rapa. BMC Genom. 2014, 15, 426. [CrossRef] [PubMed] 
46. Livak, K.J.; Schmittgen, T.D. Analysis of relative gene expression data using real-time quantitative PCR and the 2(-delta Delta C(T)) Method. Methods 2001, 25, 402-408. [CrossRef] [PubMed]

47. Amil-ruiz, F.; Garrido-Gala, J.; Blanco-portales, R.; Folta, K.M.; Munoz-Blanco, J.; Caballero, J.L. Identification and Validation of Reference Genes for Transcript Normalization in Strawberry (Fragaria $\times$ ananassa) Defense Responses. PLoS ONE 2013, 8, e70603. [CrossRef] [PubMed] 